\title{
Arctic Fog Detection Using Infrared Spectral Measurements
}

\author{
LI YI \\ Physical Oceanography Laboratory, Collaborative Innovation Center of Marine Science and Technology, and Ocean-Atmosphere \\ Interaction and Climate Laboratory, Ocean University of China, Qingdao, Shandong, China \\ KING-FAI LI \\ Department of Environmental Sciences, University of California, Riverside, Riverside, California, and Department \\ of Applied Mathematics, University of Washington, Seattle, Washington

\section{XiANYAO CHEN} \\ Physical Oceanography Laboratory, Collaborative Innovation Center of Marine Science and Technology, Ocean \\ University of China, and Qingdao National Laboratory of Marine Science and Technology, Qingdao, China \\ KA-KIT TUNG \\ Department of Applied Mathematics, University of Washington, Seattle, Washington
}

(Manuscript received 12 June 2018, in final form 23 April 2019)

\begin{abstract}
The rapid increase in open-water surface area in the Arctic, resulting from sea ice melting during the summer likely as a result of global warming, may lead to an increase in fog [defined as a cloud with a base height below $1000 \mathrm{ft}(\sim 304 \mathrm{~m})$ ], which may imperil ships and small aircraft transportation in the region. There is a need for monitoring fog formation over the Arctic. Given that ground-based observations of fog over Arctic open water are very sparse, satellite observations may become the most effective way for Arctic fog monitoring. We developed a fog detection algorithm using the temperature difference between the cloud top and the surface, called $\partial T$ in this work. A fog event is said to be detected if $\partial T$ is greater than a threshold, which is typically between -6 and $-12 \mathrm{~K}$, depending on the time of the day (day or night) and the surface types (open water or sea ice). We applied this method to the coastal regions of Chukchi Sea and Beaufort Sea near Barrow, Alaska (now known as Utqiagvik), during the months of March-October. Training with satellite observations between 2007 and 2014 over this region, the $\partial T$ method can detect Arctic fog with an optimal probability of detection (POD) between $74 \%$ and $90 \%$ and false alarm rate (FAR) between $5 \%$ and $17 \%$. These statistics are validated with data between 2015 and 2016 and are shown to be robust from one subperiod to another.
\end{abstract}

\section{Introduction}

The correlation between Arctic sea ice loss and the increase of cloud cover has been studied extensively (Eastman and Warren 2010; Intrieri et al. 2002; Kay and Gettelman 2009; Schweiger et al. 2008; Wang and Key 2005). The increase in the open-water surface area in the Arctic leads to an increase of heat and moisture exchange between the ocean and the atmosphere that favors cloud formation in nonsummer seasons (Morrison et al. 2018), which would further accelerate the sea ice

\footnotetext{
Corresponding author: King-Fai Li, king-fai.li@ucr.edu
}

loss (Cronin and Tziperman 2015; Eastman and Warren 2010; Palm et al. 2010; Shupe and Intrieri 2004; Vavrus et al. 2009). Palm et al. (2010) showed that the total cloud cover over the Arctic has increased by $\sim 0.5 \%$ per $1 \%$ decrease in sea ice extent. The reverse also holds: less cloudy (i.e., less longwave heating) winters may help recover lost sea ice, which was shown to be the case for the sea ice increase in the 2013 Arctic summer (Liu and Key 2014). However, our understanding of how the vertical cloud distribution changes is not certain. Schweiger et al. (2008) showed that using reanalysis and satellite data, the sea ice retreat during autumn was linked to a decrease in low-level cloud and an increase 
in midlevel cloud near ice margins due to an increase in surface temperature and a subsequent decrease in static stability. But Kay and Gettelman (2009) disagreed, finding more low-level cloud over open water during autumns of 2006-08 in the Arctic. Their results have been confirmed by Morrison et al. (2018) using the Cloud-Aerosol Lidar and Infrared Pathfinder Satellite Observations (CALIPSO), who suggested that the oceanatmosphere exchange of heat and moisture during autumn, and probably during other nonsummer seasons, enhances the formation of clouds over open water. There have also been indications that the occurrence of Arctic low-level cloud over open water is increasing with global warming (Eastman and Warren 2010; Palm et al. 2010).

The increase of open-water area allows new marine traffic routes in the Arctic region (Cressey 2011; Lasserre and Pelletier 2011; Stephenson et al. 2011). According to the 2013 report by the U.S. Coast Guard, $\sim 1$ million tons of freight ships traveled through the Bering Strait in 2012, which more than double the amount of ship traffic in 2008. Based on the simulated sea ice in seven climate models, Smith and Stephenson (2013) predicted that by the mid-twenty-first century, the Northwest Passage will be navigable by open-water vessels and the central Arctic will be navigable for moderate icebreakers. The increasing occurrence of fog and low stratus discussed above would greatly reduce the visibility and pose danger to aviation and shipping activities in the Arctic region (Koračin et al.2014). Historical Arctic fog and low stratus reports rely on surface observations that are widely distributed over the Eurasian continent, and are significantly less over North America and the Arctic Ocean (Eastman and Warren 2010; Warren et al. 2007). There has been spaceborne operational monitoring of fog and low stratus at high latitudes over Alaska using the Geostationary Operational Environmental Satellite-15 (GOES-15) (publicly available at https:// www.star.nesdis.noaa.gov/smcd/opdb/aviation/fog.html), but there has not been any spaceborne operational monitoring over the Arctic Ocean.

We aim to devise a satellite-based detection algorithm for near-surface clouds over the Arctic sea during the summertime. Fog and low stratus, which are very lowlevel clouds (a few hundred meters thick) near the ground, pose danger to air (especially sea planes) and marine traffic in the Arctic region (Koračin et al. 2014). However, developing robust satellite measurements for these cloud types is challenging, mainly due to the finite vertical resolution (generally much coarser than $100 \mathrm{~m}$ ) of associated spectral measurements and low thermal contrasts between surface and low-level cloud layers over polar regions. Detection of fog alone is even more difficult. The World Meteorological Organization (WMO) defines fog based on a horizontal visibility of less than $1 \mathrm{~km}$ (WMO 2005), which can hardly be used in satellite-based observations. From satellite perspectives, one detects fog from a top-down approach. Using spectral infrared and visible measurements, Bendix et al. (2005), Cermak and Bendix (2011), and Yi et al. (2016, 2015) attempted to identify fog among other cloud types in the satellite images using the zero-cloud-base-height definition, where the cloud-base height was defined by cloud-top height minus cloud thickness. The cloud thickness was estimated indirectly based on some extra knowledge such as cloud optical depth. There have also been other attempts using microphysical properties such as liquid and solid phases and cloud droplet radii to separate fog from other cloud types but the separation is not clear (see, e.g., Baldocchi and Waller 2014; Bendix et al. 2005; Cho et al. 2015). Egli et al. (2018) used the cloud-base information from ground observations to reduce the errors in satellite fog retrievals. Lidar measurements, such as the Cloud-Aerosol Lidar with Orthogonal Polarization (CALIOP) instrument aboard CALIPSO, may provide direct estimates of cloud-base heights, but Wu et al. (2015) showed that the differentiation between surface and ground-touching fog in the CALIOP measurements may be challenging.

Ellrod (2003) suggested that the cloud-top temperature of a cloud layer near the surface might be closer to the surface temperature because of a thermal inversion in the cloud than outside the cloud. Therefore, he proposed using the difference between the brightness temperature at $10.7 \mu \mathrm{m}$ and the underlying surface temperature to detect a cloud layer with a base below $1000 \mathrm{ft}$ (or $304 \mathrm{~m}$ ), which is important to aviation safety due to the fact that instrument flight rules would have to be enforced during takeoff and landing if there is a cloud layer below $1000 \mathrm{ft}$. In this paper, we shall refer to this difference as the cloud-top-sea surface temperature difference and denote it as $\partial T$ (see section 2). Ellrod (2003) was interested in both fog and low cloud. Following Lee et al. (1997), we shall treat fog and low cloud as one meteorological object and we refer to such an object to as "fog/low cloud." The $\partial T$ method has been adopted in several studies (Gultepe et al. 2007; Wilcox 2017; Wu and Li 2014) as an initial step to identify fog/lowcloud scenes at midlatitudes, which are then further separated into fog scenes and low cloud scenes with the help of other constraints (e.g., brightness temperature at $3.7 \mu \mathrm{m}$ ). Application of this method to the polar region is much more challenging and so far there has not been any reported study. Our goal of this work is to demonstrate that the $\partial T$ method can also be used to identify fog/low cloud at polar latitudes. Further separation of fog and low cloud at polar latitudes, however, is beyond our scope of understanding and will be deferred to future publications. 
While lidar measurements provide direct measurements of cloud-base height for defining fog/low cloud, we emphasize that infrared-based detection, such as the $\partial T$ method, offers a few advantages: 1) Lidar measurements only allow strictly downward light path (i.e., $0^{\circ}$ at nadir) with a swath width of order $100 \mathrm{~m}$. Therefore the spatial coverage of the lidar measurements is sparse and is not ideal for operational weather monitoring. In contrast, infrared measurements, such as the Moderate Resolution Imaging Spectroradiometer (MODIS), have wide across-track scanning geometry (of order $1000 \mathrm{~km}$ ) that provides spatiotemporally dense coverage of the globe [see Fig. 1 of Holz et al. (2008) for a comparison of the collocated and CALIPSO pixels]. Figure 1 shows the $\sim 30$ swaths of the A-Train (which includes CALIPSO and MODIS in its constellation) on 1 June 2018, which converge toward the pole. The wide across-track MODIS scans provide a full coverage of the polar region $\sim 30$ times per day; 2) there are currently a number of spaceborne infrared measurements [such as the GOES series, the Atmospheric Infrared Sounder (AIRS) and Infrared Atmospheric Sounding Interferometer (IASI)] that can be used to cross validate the fog detection algorithm; and 3) historical infrared measurements date back to late 1970s [by the measurements of NOAA's Advanced Very High Resolution Radiometer (AVHRR)], so that Arctic fog/low cloud in the past few decades may also be estimated using these historical measurements.

The $\partial T$ method relies on a threshold to define the presence of fog/low cloud. In the search of the threshold algorithm, Ellrod (2003) used surface-based observations as prior information to define fog/low-cloud scenes in the satellite images. However, surface observations over the Arctic Ocean are scarce. Instead, we will use the cloud-base height measurements by CALIPSO as prior information to define fog/low-cloud scenes for the purpose of algorithm development. The $\partial T$ thresholds will be derived using the MODIS along-track pixels that are collocated with the $C A L I P S O$ pixels at $0^{\circ}$ nadir. Due to the significantly different footprint geometries (MODIS's $1 \mathrm{~km}$ versus CALIPSO's $333 \mathrm{~m}$ ) and the orbital time gap ( $\sim 73 \mathrm{~s})$ between MODIS and CALIPSO, cloud structures (vertical or horizontal) in the field of views of the collocated pixels may also be different. Therefore, we must carefully select the MODIS and CALIPSO pixels to ensure that both instruments "see" the same cloud. The details of data selection will be described in section 2c. Once these $\partial T$ thresholds are derived, they can then be applied generally for fog/lowcloud detection to regions where there are cloud-top temperature measurements only, such as the MODIS across-track pixels. A shortcoming of our fog/low-cloud

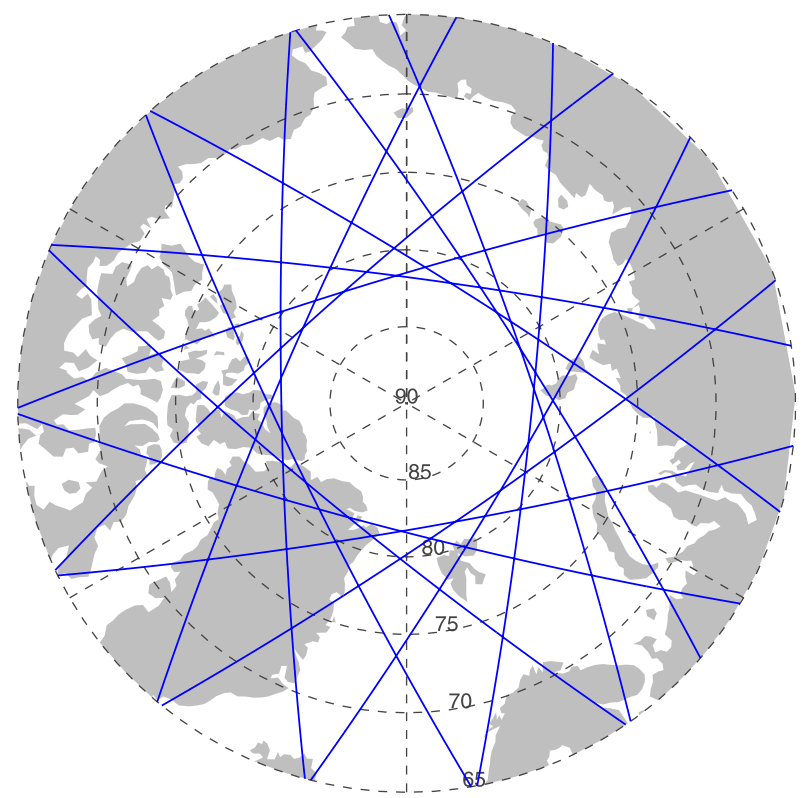

FIG. 1. The A-Train swath paths (blue lines) over the Arctic on 1 June 2018. The MODIS and CALIPSO instruments are members of the A-Train satellite constellation.

detection algorithm is that MODIS or other infrared measurements cannot probe through multilayered clouds. Also, the CALIPSO's lidar signal that we use for validation is strongly attenuated through thick clouds. Therefore, the verification statistics against CALIPSO can be derived only when single-layered clouds are present. For multilayered clouds, the false alarm rate (FAR) may be higher than the nominal value $(5 \%-17 \%)$ to be derived in this study, but is unknowable at present.

The rest of the paper is organized as follows. In section 2, we describe an Arctic fog/low-cloud detection method and describe the datasets to be used for the detection. Section 3 presents our derivation of the $\partial T$ thresholds over four different polar scenarios. An example of fog/lowcloud detection based on the $\partial T$ method is also presented. Comparison with available cruise in situ measurements is also provided. Section 4 summarizes our results.

\section{Data and method}

\section{a. $\partial T$ definition}

Ellrod's (2003) infrared-based $\partial T$ method will be modified for the Arctic environment and for day as well as night time fog/low-cloud detection in this paper. We follow Zhang and Yi (2013) to define $\partial T$ as cloud-top temperature minus surface temperature:

$$
\partial T=\text { cloud-top temperature }- \text { surface temperature }
$$


Equation (1) differs from Ellrod's (2003) definition by a negative sign. According to Ellrod (2003), a cloud object over the United States is considered fog/low cloud if the surface temperature is not $4 \mathrm{~K}$ warmer than the cloudtop temperature. Therefore, the $\partial T$ threshold is $-4 \mathrm{~K}$ according to Eq. (1) in Ellrod's (2003) case: fog/low cloud is said to be detected if $\partial T \geq-4 \mathrm{~K}$. Ellrod (2003) applied the $\partial T$ method for nighttime fog/low-cloud detection. Zhang and $\mathrm{Yi}(2013)$ showed that the $\partial T$ method is applicable to the Yellow Sea at midlatitudes, during the daytime, with a season-dependent $\partial T$ threshold ranging from -3 to $-4.5 \mathrm{~K}$. With modifications to the detection threshold, we will show that the $\partial T$ method can also detect fog/low cloud during the Arctic daytime over ocean and ice surfaces.

Zhang and Yi (2013) also showed that instead of using instantaneous sea surface temperature, the climatological mean of the sea surface temperature may serve to detect sea fog with satisfactory accuracy, thereby greatly simplifying the detection algorithm. To carry out a study about fog/low-cloud changes over the Arctic, in contrast, we need a time varying sea surface temperature to account for year-to-year variations in sea ice cover. Therefore, in this work, we will use MODIS's sea surface temperature to increase the accuracy of the fog/low-cloud detection.

\section{b. MODIS data}

Ellrod (2003) used the GOES measurements of the $10.7-\mu \mathrm{m}$ brightness temperature as a proxy for the cloud-top temperature. Here, we use channel 31 (10.78$11.28 \mu \mathrm{m})$ brightness temperature measured by the MODIS (Ackerman et al. 1998) aboard the Aqua satellite platform as the proxy of the cloud-top temperature. MODIS channel 31 has a spatial resolution of $1 \mathrm{~km}$. The MODIS across-track swath width is $2030 \mathrm{~km}$.

The period to be studied includes the months March to October from 2007 to 2016. MODIS collection 6 data are used. The geolocation information and the solar zenith angle in the MYD03 product are used. The solar zenith angle is used to determine the day (when $|\mathrm{SZA}| \leq 90^{\circ}$ ) or night (when $|\mathrm{SZA}| \geq 90^{\circ}$ ) of the measurement. The brightness temperature of channel 31 is obtained from the level 1 MYD021KM product. Cloud-top height (to be used in sections $2 \mathrm{c}$ and $3 \mathrm{c}$ ) and surface temperature are obtained from the level 2 MYD06 product. The unobstructed field-of-view (FOV) quality flag in the level 2 MYD35 cloud mask product takes four possible values ( 0 for confident cloudy, 1 for possibly cloudy, 2 for possibly clear, and 3 for confident clear); we only use those MODIS footprints that have a zero unobstructed FOV quality flag as cloudy scenes.

Over the Arctic Ocean, there may be areas of sea ice adjacent to areas of open water. In our calculations, we use the four-times-daily reanalysis sea surface temperature data (TMP:sfc) provided in the MYD06 product to define open-water and sea ice regions. An open-water region is defined when the MODIS surface temperature is above $271.35 \mathrm{~K}$ (or, equivalently, $-1.8^{\circ} \mathrm{C}$ ) while a sea ice region is defined when the MODIS surface temperature is below 271.35 K (Reynolds et al. 2007). (Figure 3 lists the MODIS datasets that are involved in the fog detection.)

\section{c. Prior fog/low-cloud knowledge from CALIPSO}

The CALIPSO level 2 vertical feature mask (VFM) product (CAL_LID_L2_VFM-Standard-V4-10) provides the geolocation information (longitude, latitude, and altitude) along the swath path. Each nadir footprint has a diameter of $330 \mathrm{~m}$. Below $8.2 \mathrm{~km}$, the vertical resolution of the VFM product is $30 \mathrm{~m}$; between 8.2 and $20.2 \mathrm{~km}$, the vertical resolution in $60 \mathrm{~m}$. Each layer is classified by the variable "feature type" in the VFM product as "clear," "cloudy," "aerosol," "stratospheric feature," "surface," "subsurface," or "no signal."

Given a MODIS cloudy scene at nadir (i.e., $0^{\circ}$ viewing angle), there are 3 CALIPSO $330-\mathrm{m}$ footprints that overlap with the MODIS 1-km footprint. We pick the CALIPSO footprint closest to the center of the MODIS footprint and use the corresponding VFM product to check whether the MODIS cloudy scene is also a CALIPSO cloudy scene. Another strategy is to take the average of the VFM products of all three CALIPSO footprints to define a CALIPSO cloudy scene. We tested both strategies and verified that the conclusions remain unchanged. We ignored the CALIPSO scenes where there is at least one aerosol layer in the VFM product. Therefore, we only pick aerosol-free CALIPSO footprints. The cloud-top height of a CALIPSO cloudy scene is defined by the altitude of the highest VFM layer that contains a cloud.

Both MODIS and CALIPSO are members of the A-Train constellation and they both cross the equator at around 0130 and 1330 LT, with MODIS (on Aqua) $\sim 73 \mathrm{~s}$ ahead of CALIPSO. Due to differences in the measurement techniques and geometries, prescreening of the collocated MODIS and CALIPSO pixels is required to make sure that the observations of the two instruments are inherently consistent with each other, as discussed in section 1 . First, we require that the cloud object in the field of view must be single layered to avoid undetected high clouds that may offset the MODIS cloud-top temperatures. This screening is achieved by using CALIPSO's lidar measurement, which is capable of detecting more than one cloud layers in the field of view. Two or more cloudy levels, adjacent to each other, are considered to form one continuous cloud layer. A CALIPSO scan is said to detect a single-layered cloud, 


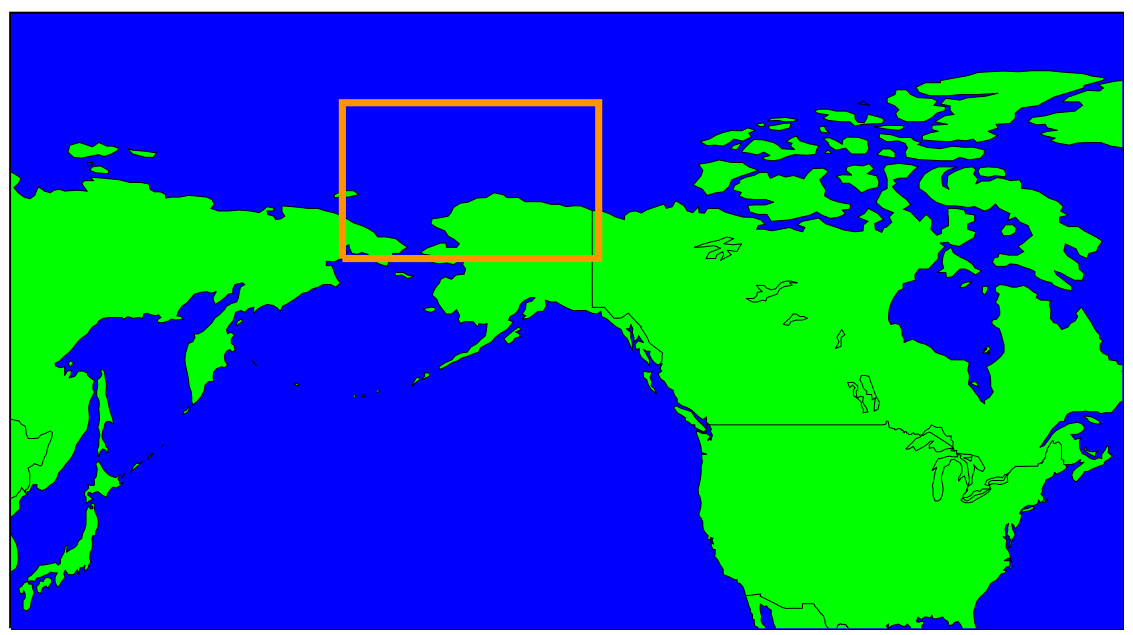

FIG. 2. The region over the Beaufort Sea and the Chukchi Sea (enclosed by the rectangle) where collocated MODIS and CALIPSO cloud observations in the extended summer (MarchOctober) during 2007-14 are used in this study.

if there is only one continuous cloud layer. In addition, if the single-layered cloud is optically thick (optical depth $\geq 3$ ), then the CALIPSO retrieval product returns "no signal" below this cloud due to the strong lidar attenuation. These cases are also excluded in the collocation process in order to avoid missing fog scenes underneath thick clouds.

Second, we require that the cloud-top heights from MODIS and CALIPSO agree with each other to eliminate pixels containing cloud edges, especially those of small high clouds over large low clouds that MODIS is not able to resolve. Cloud-top height is one of very few variables that are reported by both MODIS and CALIPSO, which we can use for direct comparison. However, since MODIS does not measure the cloud-top height directly, the estimated cloud-top heights may be subject to large errors, especially for very low-level clouds. Early investigations suggest that the MODIS cloud-top height for low cloud $(\leq 4 \mathrm{~km})$ in the Arctic region was biased high against the $C A L I P S O$ cloud-top height by $0.3 \mathrm{~km}$ (Holz et al. 2008). For low clouds (below $700 \mathrm{hPa}$ ), the MODIS cloud-top height is converted from the MODIS cloud-top temperature (determined from channel 31), which requires reanalysis temperature profiles. In pixels where temperature inversion occurs (which is the common case for fog formation), the MODIS cloud-top height is assigned the same temperature level above the inversion layer rather than the true cloud-top height (Menzel et al. 2008), leading to a significant margin of error in the MODIS cloud-top height determination under polar conditions. Thus, collocated MODIS and CALIPSO observations will be used only if the following heuristic criterion is satisfied:

$$
\left|H_{\text {MODIS }}-H_{\text {CALIPSO }}\right| \leq a H_{\text {MODIS }}+b \text {, }
$$

where $a=20 \%$ and $b=0.2 \mathrm{~km}$. In other words, we select the MODIS observation only if the measured cloudtop height agrees with the CALIPSO cloud-top height within $20 \%$. A larger $a$ allows more collocated data to be used but the discrepancy between the observed MODIS and CALIPSO cloud structures would also be larger. The constant $b$ is meant to offset the MODIS bias. Note that a $0.2-\mathrm{km}$ bias for high cloud-top heights may represent a few percent of error only but a $0.2-\mathrm{km}$ bias for low cloud-top heights may represent a few dozen percent. Therefore, high clouds are not sensitive to $b$ but low clouds are sensitive to $b$. Conversely, a $20 \%$ error in the low cloud-top heights may be a few hundred meters only but a $20 \%$ error in the high cloud-top heights may be a few kilometers. Therefore, low clouds are not sensitive to $a$, but high clouds are sensitive to $a$. We have tried several values of $a$ and $b$; the current values of $a$ and $b$ are optimal choices such that about $20 \%$ of the collocated MODIS-CALIPSO measurements are used for the subsequent $\partial T$ assessments.

We used CALIPSO data for the period 2007-14 for training and reserved 2015/16 for validation. We collect the collocated MODIS and CALIPSO footprints over the coastal regions of the Chukchi and the Beaufort Seas during the period March-October from 2007 to 2014 (Fig. 2). The $\partial T$ values corresponding to the collocated footprints are divided into "fog/low cloud" and "other cloud" groups using the CALIPSO cloud-base heights. The "fog/low cloud" group corresponds to cloud-base heights less than $1000 \mathrm{ft}$ and the "other cloud" group corresponds to cloud-base heights greater than $1000 \mathrm{ft}$. 


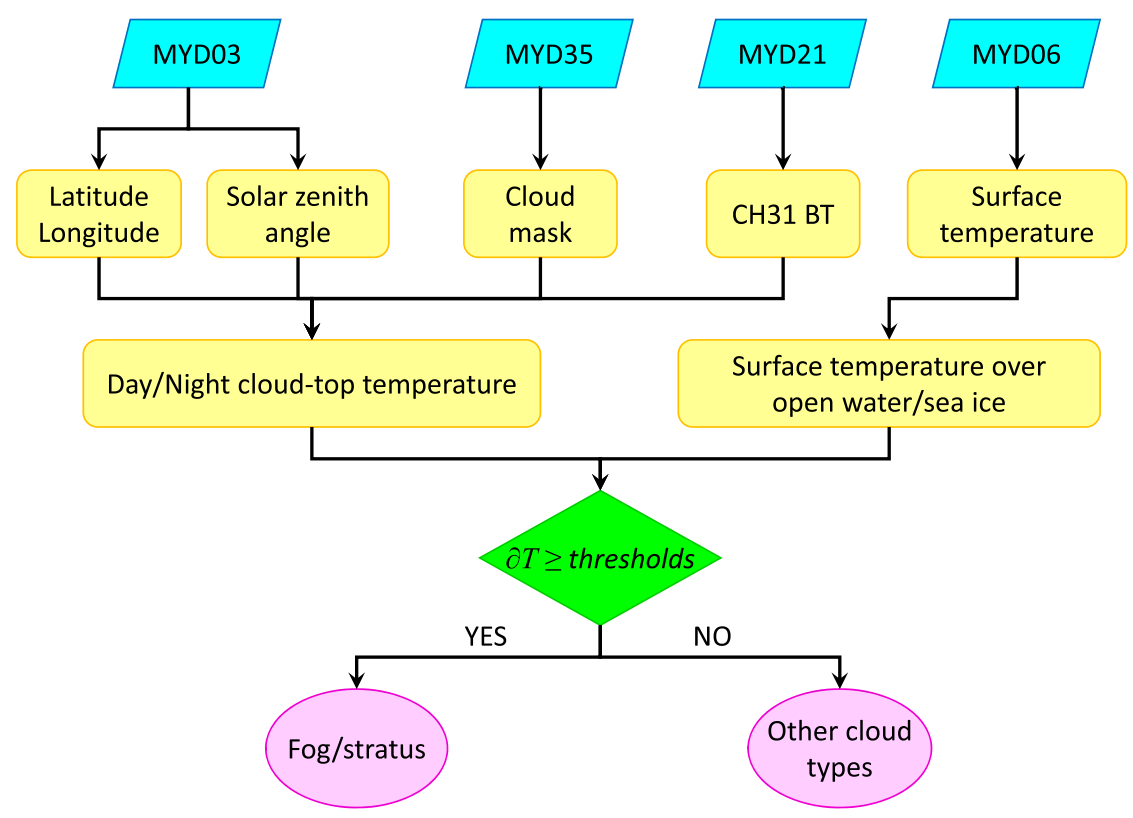

FIG. 3. A flowchart of the Arctic fog detection algorithm.

The histograms of the $\partial T$ values in these two groups are obtained (cf. Fig. 4).

\section{d. The proposed fog/low-cloud detection algorithm}

A flowchart of the Arctic fog/low-cloud detection using MODIS data is shown in Fig. 3: we first check whether the observed scene is cloudy from the cloud mask. Then we determine the day and night periods using the solar zenith angle information and the surface type (open water or sea ice) using MODIS surface temperature. Last, we calculate the $\partial T$ value using MODIS cloud-top temperature and surface temperature.

\section{Results and discussion}

\section{a. Derivation of the $\partial T$ thresholds with training data between 2007 and 2014}

Below, we will derive different $\partial T$ thresholds for different polar conditions.

We will analyze the $\partial T$ distributions for two time periods (nighttime and daytime observations) and over two surface types (open water and sea ice). The division between nighttime and daytime periods is to confirm if the detection based on the infrared channel should be insensitive to the presence of sunlight. The division between open-water and sea ice regions is to test whether our method is affected by the surface types.

\section{1) First SCENARIO: DAYTIME OVER OPEN WATER}

We begin the analysis with the MODIS daytime (when solar zenith angle is less than $90^{\circ}$ ) data over open water. This is the most relevant situation for summer open-water navigation. The corresponding the $\partial T$ data will be denoted by $\partial T_{\text {day/water }}$.

As explained in section 2c, a subset of MODIS footprints is carefully selected to match with CALIPSO's. Of the observed $\partial T_{\text {day/water values, } 114622 \text { are classified }}$ into the "fog/low cloud" group (i.e., CALIPSO cloudbase height $\leq 1000 \mathrm{ft}$ ) and 168434 of them are classified into the "other cloud" group. Figure 4a shows the distributions of $\partial T_{\text {day/water }}$ in these two groups on 2-K bins. The $\partial T_{\text {day/water }}$ distribution for fog/low cloud is slightly negatively skewed, ranging from -10 to $4 \mathrm{~K}$ with a peak at $-3 \mathrm{~K}$. The $\partial T_{\text {day/water }}$ distribution for the "other cloud" group ranges from -40 to $-2 \mathrm{~K}$ with a peak at $-13 \mathrm{~K}$.

Figure $4 \mathrm{a}$ thus suggests that the $\partial T_{\text {day/water }}$ threshold should lie between the peaks of the two distributions (i.e., between -3 and $-13 \mathrm{~K}$ ). To search for the threshold quantitatively, we have tried several thresholds along the $x$ axis in Fig. 4a and picked the threshold that "best" identifies the fog/low-cloud scenes. The sensitivity of the result to the choice of the threshold is discussed below, where the errors are quantified using two verification statistics.

The $\partial T$ method detects the presence of fog/low cloud as follows: on the right-hand side of the threshold, the MODIS cloudy scenes will be deemed as "fog/low cloud"; on the left-hand side of the threshold, all MODIS cloudy scenes will be defined as "other cloud." Following Schaefer (1990), we define the following cases:

Case $X$ : The cloudy scene is a MODIS fog/low-cloud case (i.e., $\partial T$ lies on the right-hand side of the 

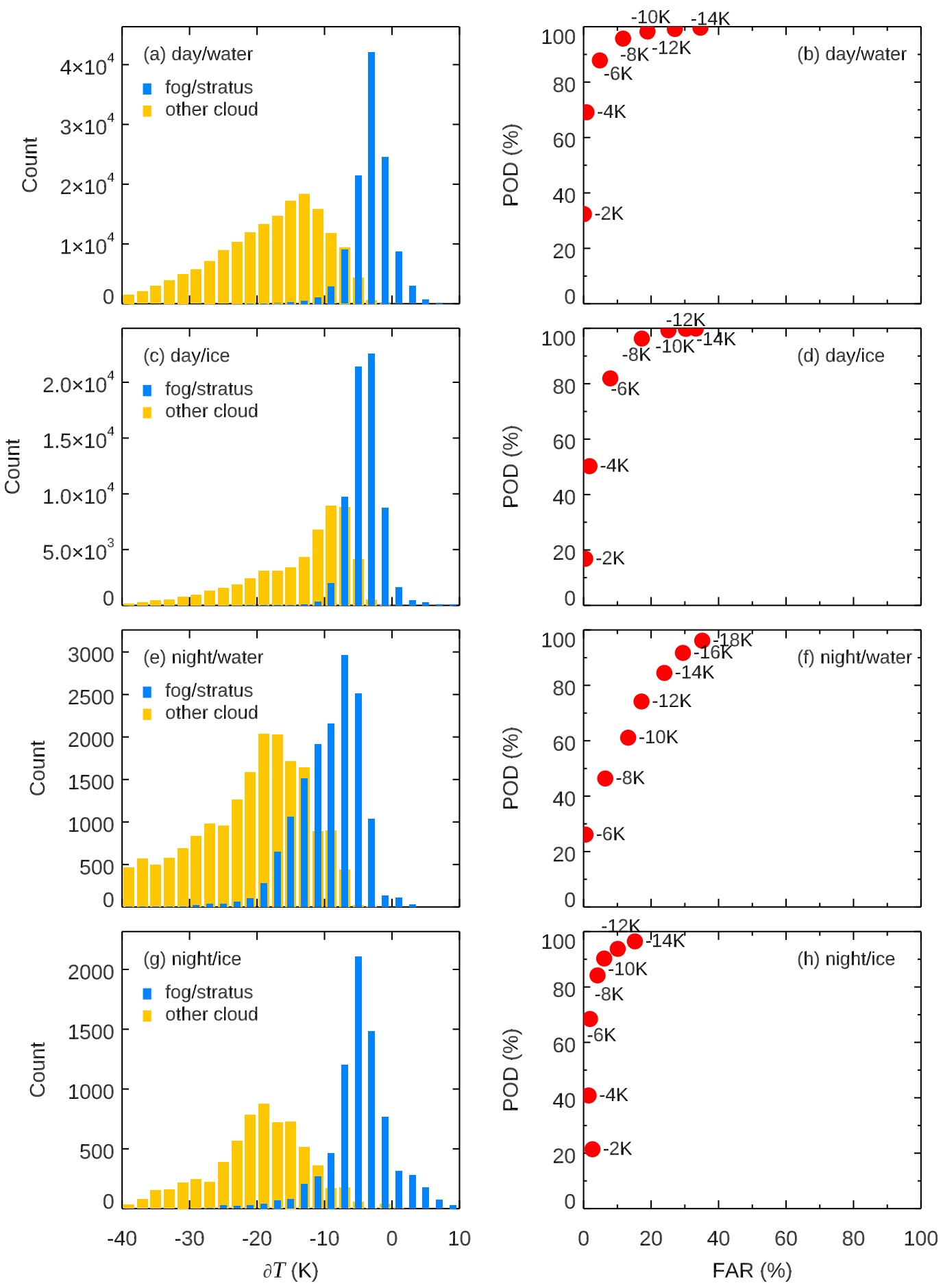

FIG. 4. (a) The distribution of the cloud-top-sea surface temperature difference $\partial T$ for fog (with a base below $1000 \mathrm{ft}$ or $304 \mathrm{~m}$; blue vertical bars) and nonfog (i.e., other cloud types; yellow vertical bars) during daytime over

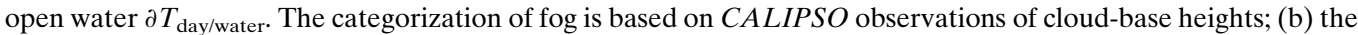
probability of detection (POD) and false alarm rate (FAR) as a function of the trial $\partial T$ threshold values. The number next to each dot is the corresponding threshold value. Points lying in the top-left quadrant may be selected

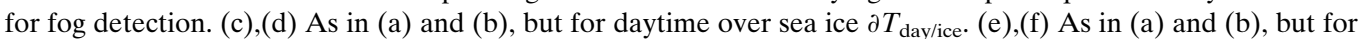
nighttime over open water $\partial T_{\text {night/water. }}(\mathrm{g}),(\mathrm{h}) \mathrm{As}$ in (a) and (b), but for nighttime over sea ice $\partial T_{\text {day/ice. }}$. 
threshold) and it is also a CALIOP fog/low-cloud case. Thus the $\partial T$ method reports a correct positive.

Case $Y$ : The cloudy scene is a not MODIS fog/lowcloud case (i.e., $\partial T$ lies on the left-hand side of the threshold) but it is a CALIOP fog/low-cloud case. Thus the $\partial T$ method reports a false negative.

Case $Z$ : The cloudy scene is a MODIS fog/low-cloud case but it is a not CALIOP fog/low-cloud case. Thus the $\partial T$ method reports a false positive (also known as false alarm).

Case $W$ : The cloudy scene is not a MODIS fog/lowcloud case nor a CALIOP fog/low-cloud case. Thus the $\partial T$ method reports a correct negative.

Two verification statistics are used to test the performance of the threshold. The first one is the probability of detection (POD):

$$
\mathrm{POD}=\frac{X}{X+Y},
$$

which is simply the fraction of "real" (seen by CALIPSO) fog/low-cloud cases that are successfully identified by MODIS. The more negative the threshold, the closer the POD is to $100 \%$. For example, based on Fig. 4a, the POD would be $99 \%$ if the threshold were to be $-14 \mathrm{~K}$ and all real fog/low-cloud cases (the cyan bars in Fig. 4a) would be captured. However, this $99 \%$ POD is misleading because a large portion of other cloud (the yellow bars in Fig. 4a) is also misidentified as fog/low cloud. Therefore, we need another verification quantity, the FAR:

$$
\mathrm{FAR}=\frac{Z}{X+Z}
$$

which is the fraction of "detected" (seen by MODIS) fog/low-cloud cases that are not real fog/low cloud (seen by CALIPSO); the more negative the threshold, the larger is FAR. In the previous example, if the threshold were to be $\partial T_{\text {day/water }} \geq-14 \mathrm{~K}$, which yields a $99 \%$ POD, the FAR is $35 \%$, a false alarm rate that is not acceptable. A practical threshold is a compromise that would result in a high POD while maintaining a low FAR.

One may also compute the critical success index (CSI; also known as the threat rate) and the bias score, defined, respectively, as

$$
\mathrm{CSI}=\frac{X}{X+Y+Z},
$$

and

$$
\text { Bias score }=\frac{X+Z}{X+Y}
$$

TABLE 1. Recommended $\partial T$ thresholds for Arctic fog detection in four scenarios: daytime over open water, daytime over sea ice, nighttime over open water, and nighttime over sea ice. The verification statistics, POD, FAR, CSI, and bias, corresponding to each scenario are also tabulated.

\begin{tabular}{lccccc}
\hline \hline Scenario & $\begin{array}{c}\text { Threshold } \\
(\mathrm{K})\end{array}$ & $\begin{array}{c}\text { POD } \\
(\%)\end{array}$ & $\begin{array}{c}\text { FAR } \\
(\%)\end{array}$ & $\begin{array}{c}\text { CSI } \\
(\%)\end{array}$ & $\begin{array}{c}\text { Bias } \\
(\%)\end{array}$ \\
\hline $\begin{array}{l}\text { Day/open } \\
\text { water }\end{array}$ & -6 & 88 & 5 & 84 & 92 \\
$\begin{array}{l}\text { Day/sea ice } \\
\text { Night/open }\end{array}$ & -6 & 82 & 8 & 77 & 89 \\
$\quad$ water & -12 & 74 & 17 & 64 & 90 \\
Night/sea ice & -10 & 90 & 6 & 85 & 96 \\
\hline
\end{tabular}

The CSI measures the fraction of observed or forecast events that are correctly predicted, which is given by the fraction of MODIS successful detection of fog/low cloud among all cases where MODIS or CALIPSO, or both, report a fog/low cloud. The bias score measures the ratio of the frequency of forecast events to the frequency of observed events, which is given by the number of MODIS fog/low-cloud events over the number of CALIPSO fog/low-cloud events. Both the CSI and the bias score should be close to $100 \%$ for an optimal detection.

Figure $4 \mathrm{~b}$ plots the values of POD against FAR as a function of the threshold in the range -14 to $-2 \mathrm{~K}$. As mentioned above, at $-14 \mathrm{~K}$, the POD is $99 \%$ but the FAR is $35 \%$. The FAR can be reduced to $1 \%$ when the threshold is $-4 \mathrm{~K}$, but then the POD is only $69 \%$. To achieve a high POD and a low FAR, an appropriate threshold corresponds to a point located near the upperleft corner of the $\Gamma$-shaped POD-FAR curve. Deviations (of order $\pm 1 \mathrm{~K}$ ) around this corner would lead to either a large decrease POD (if a less negative threshold is chosen) or a large increase in FAR (if a more negative threshold is chosen). Therefore, we recommend the threshold $\partial T_{\text {day/water }} \geq-6 \mathrm{~K}$, whence the POD is $88 \%$ and the FAR is $5 \%$ (Table 1). In words, if the threshold were $\partial T_{\text {day/water }} \geq-6 \mathrm{~K}$, then $88 \%$ of all fog/lowcloud events would be successfully detected. And if our detection algorithm says, "there is fog/low cloud," then such a detection has a probability of $5 \%$ that the "suspected fog/low cloud" may turn out to be other cloud. With this threshold, the CSI is $84 \%$ and the bias score is $92 \%$.

In Eq. (2), if $a$ is varied between $10 \%$ and $30 \%$, then the POD and FAR would vary between $85 \%$ and $88 \%$ and between $3 \%$ and $6 \%$, respectively, for the threshold $\partial T_{\text {day/water }} \geq-6 \mathrm{~K}$. If $b$ were varied between 100 and $300 \mathrm{~m}$, then the POD and FAR would vary between $86 \%$ and $88 \%$ and between $5 \%$ and $6 \%$, respectively. However, in both perturbation tests, the $\Gamma$-shaped 
TABLE 2. A robustness test of the recommended $\partial T$ thresholds with the MODIS-CALIPSO collocated data. The test has been performed by applying the $\partial T$ thresholds (cf. Table 1$)$ to 2 -yr segments $(2007 / 08,2009 / 10,2011 / 12$, and 2013/14) of the total record.

\begin{tabular}{|c|c|c|c|c|c|c|}
\hline Scenario & Threshold (K) & Segment & POD (\%) & FAR (\%) & CSI (\%) & Bias (\%) \\
\hline \multirow[t]{4}{*}{ Day/open water } & -6 & $2007 / 08$ & 89.23 & 4.70 & 85.47 & 93.63 \\
\hline & & $2009 / 10$ & 90.09 & 3.66 & 87.11 & 93.52 \\
\hline & & $2011 / 12$ & 86.77 & 3.54 & 84.09 & 89.95 \\
\hline & & $2013 / 14$ & 84.87 & 7.99 & 79.05 & 92.25 \\
\hline \multirow[t]{4}{*}{ Day/sea ice } & -6 & $2007 / 08$ & 79.82 & 4.82 & 76.72 & 83.86 \\
\hline & & $2009 / 10$ & 81.94 & 5.93 & 77.91 & 87.10 \\
\hline & & $2011 / 12$ & 82.83 & 9.81 & 75.99 & 91.84 \\
\hline & & $2013 / 14$ & 82.75 & 10.42 & 75.48 & 92.38 \\
\hline \multirow[t]{4}{*}{ Night/open water } & -12 & $2007 / 08$ & 69.93 & 27.33 & 55.37 & 96.22 \\
\hline & & $2009 / 10$ & 66.87 & 9.21 & 62.62 & 73.65 \\
\hline & & $2011 / 12$ & 77.21 & 8.70 & 71.92 & 84.58 \\
\hline & & $2013 / 14$ & 77.40 & 27.48 & 59.85 & 106.73 \\
\hline \multirow[t]{4}{*}{ Night/sea ice } & -10 & $2007 / 08$ & 89.20 & 9.58 & 81.50 & 98.65 \\
\hline & & $2009 / 10$ & 93.63 & 9.05 & 85.65 & 102.95 \\
\hline & & $2011 / 12$ & 83.33 & 5.14 & 79.74 & 87.85 \\
\hline & & $2013 / 14$ & 93.51 & 2.23 & 91.55 & 95.64 \\
\hline
\end{tabular}

curve remains unchanged and $\partial T_{\text {day/water }} \geq-6 \mathrm{~K}$ remains as an optimal choice of the threshold. This holds true for other polar conditions discussed in sections $3 a(2)-(4)$. Therefore, in the rest of the paper, we only discuss the thresholds determined using $a=20 \%$ and $b=0.2 \mathrm{~km}$.

The statistics obtained above can be shown to be robust over different periods: We divided the MODIS/CALIPSO collocated data into four equal segments spanning from 2007 to 2008, from 2009 to 2010 , from 2011 to 2012, and from 2013 to 2014. We applied the $\partial T \geq-6 \mathrm{~K}$ threshold that has been derived above to define fog/low cloud in each segment. The resultant POD, FAR, CSI and the bias score are shown in Table 2. The means of POD, FAR, CSI and the bias score of the five segments are the same as those statistics in Table 1. The values of the POD, FAR, CSI and the bias score vary between $82 \%$ and $90 \%$, between $3 \%$ and $8 \%$, between $79 \%$ and $87 \%$, and between $85 \%$ and $94 \%$, respectively. Therefore, the uncertainties of the POD, FAR, CSI and the bias score are of order only a few percent.

\section{2) DAYTIME OVER SEA ICE}

The above analysis can be easily generalized to other periods and surface types. Figure $4 \mathrm{c}$ shows the $\partial T$ distributions during daytime over sea ice. The corresponding $\partial T$ data will be denoted by $\partial T_{\text {day/ice }}$. There are 67566 fog/low-cloud scenes and 53644 other cloud scenes. The total number of MODIS observations in this scenario is significantly less than that over open water because there is less sea ice during the summertime.
The $\partial T_{\text {day/ice }}$ distribution is predominantly between -10 and $2 \mathrm{~K}$ for fog/low cloud and between -30 and $-4 \mathrm{~K}$ for other cloud. Based on Fig. 4d, we suggest the threshold $\partial T_{\text {day } / \text { ice }} \geq-6 \mathrm{~K}$, whence the POD is $82 \%$, the FAR is $8 \%$, the CSI is $77 \%$, and the bias score is $89 \%$ (Table 1). Similar to the previous scenario, the uncertainties of the verification statistics are of order a few percent (Table 2).

\section{3) NightTIME OVER OPEN WATER}

Figure $4 \mathrm{e}$ shows the $\partial T$ distributions during nighttime over open water $\partial T_{\text {night/water }}$. There are $14629 \mathrm{fog} / \mathrm{low}-$ cloud scenes and 19982 other cloud scenes. Note that the total number of observations during nighttime is much less than that during the daytime because the criteria for "nighttime" retrievals occur less often from March to October than the criteria for "daytime" retrievals. Unlike the scenarios during the daytime, the $\partial T_{\text {night/water }}$ distribution for fog/low cloud during the nighttime ranges between -20 and $4 \mathrm{~K}$, with a peak at $-9 \mathrm{~K}$. The $\partial T_{\text {night/water }}$ distribution for other cloud is ranged between -50 and $-4 \mathrm{~K}$, with a peak at $-19 \mathrm{~K}$. Thus, there is significant overlap between the distributions of the two groups. Furthermore, the curvature of the POD-FAR curve is much less obvious in Fig. 4f. The FAR stays above $20 \%$ for most valid POD values. As a result, we suggest the threshold $\partial T_{\text {night/water }} \geq-12 \mathrm{~K}$, whence the POD is $74 \%$, the FAR is $17 \%$, the CSI is $64 \%$, and the bias score is $90 \%$ (Table 1 ). The uncertainties of the verification statistics for this scenario are the largest among all scenarios, which are of order $10 \%-25 \%$, due to the shorter nighttime and hence the smaller sample in the summer. 
TABLE 3. The verification statistics when the recommended $\partial T$ thresholds listed in Table 2 are applied to the MODIS-CALIPSO collocated data in March-October during 2015/16.

\begin{tabular}{lccccrr}
\hline \hline \multicolumn{1}{c}{ Scenario } & Threshold (K) & Segment & POD (\%) & FAR (\%) & CSI (\%) & Bias (\%) \\
\hline Day/open water & -6 & $2015 / 16$ & 82.18 & 4.12 & 79.37 & 85.71 \\
Day/sea ice & -6 & $2015 / 16$ & 73.93 & 5.06 & 71.13 & 77.87 \\
Night/open water & -12 & $2015 / 16$ & 87.27 & 9.01 & 80.33 & 95.91 \\
Night/sea ice & -10 & $2015 / 16$ & 95.45 & 4.88 & 90.99 & 100.35 \\
\hline
\end{tabular}

\section{4) NightTime OVER SEA ICE}

The last scenario to be examined is the $\partial T$ distribution during nighttime over sea ice $\partial T_{\text {night/ice. }}$. Figure $4 \mathrm{~g}$ shows the $\partial T_{\text {night/ice }}$ distribution. There are 7626 fog/low-cloud scenes and 6630 other cloud scenes. The $\partial T_{\text {night/ice }}$ distribution for fog/low-cloud covers from -20 to $10 \mathrm{~K}$, with a peak at $-1 \mathrm{~K}$. The $\partial T_{\text {night/ice }}$ distribution for other cloud mostly covers from -30 to $2 \mathrm{~K}$, with a peak $-11 \mathrm{~K}$. Based on Fig. $4 \mathrm{~h}$, the threshold we suggest for this scenario is $\partial T_{\text {night/ice }} \geq-10 \mathrm{~K}$, whence the POD is $90 \%$, the FAR is $6 \%$, the CSI is $85 \%$, and the bias score is $96 \%$ (Table 1). The uncertainties of the verification statistics are of order $5 \%-10 \%$ (Table 3).

\section{5) VALidation WITH THE 2015/16 MODIS AND CALIPSO DATA}

To validate the $\partial \mathrm{T}$ method, we apply the thresholds to the MODIS data acquired in 2015/16 and recompute the verification statistics (POD, FAR, CSI, and bias) using the corresponding MODIS/CALIPSO collocated data, in the same way that we derived the thresholds. These MODIS data (and the MODIS/ CALIPSO collocated data) were not involved the derivation of the thresholds. Therefore, the resultant verification statistics (shown in Table 3) serve as an independent validation of our fog detection algorithm. Among the four scenarios, the POD ranges between $75 \%$ and $100 \%$, the FAR ranges between $7 \%$ and $20 \%$, the CSI ranges between $70 \%$ and $86 \%$, and the bias ranges between $84 \%$ and $126 \%$. These PODs, FARs, CSIs, and biases are consistent with those listed in Tables 1 and 2, showing that the fog detection algorithm is reliable.

\section{b. A case study}

We have applied the algorithm to a MODIS scene over the Beaufort Sea region. Figure 5 shows the swath path of MODIS over the Arctic at 2305 UTC 15 July 2016. An observed scene is fog/low cloud if the $\partial T$ value is greater than the thresholds listed in Table 1. A visible image of the cloud system is shown in Fig. 5a. Figure $5 b$ is an infrared map of detected fog/low cloud (gray shades) and other cloud types (yellow shades) using the $\partial T$ method. The cyan line in both panels shows the coastlines in the region. For comparison, fog/low cloud (pink; cloud-base height $\leq 304 \mathrm{~m}$ ) and other cloud types (light green) inferred from CALI$P S O$ is overlaid. Note that the actual swath width of CALIPSO is much smaller than the line width shown in Fig. 5. Also note that the total swath width of MODIS is much wider than that of CALIPSO. Along the track, the fog/low-cloud pixels obtained using the $\partial T$ method agree well with those obtained from CALIPSO. In the visible image (Fig. 5a), highly reflective, white pixels are generally associated with thick low/middle clouds and are identified as other cloud types in the infrared image (Fig. 5b). Most other white pixels are identified fog/low cloud in the infrared image. However, there are white pixels in the visible image that appears as blue in the infrared image. These pixels are clear sky, with sea ice at the ocean surface. In these circumstances, we assign "ocean" in Fig. 5b for visualization.

\section{c. Comparing with the traditional bispectral method}

The bispectral method has been used to detect the presence of fog/low cloud at midlatitudes (Cermak and Bendix 2007; Gao et al. 2009; Hunt 1973; Yamanouchi and Kawaguchi 1992) based on the cloud-top information. This method works the best for nighttime cloud detection when there is no contamination by scattered sunlight in the near-infrared channel at $3.9 \mu \mathrm{m}$ (Lee et al. 1997). Lee et al. (1997) suggested in their Fig. 1 that when fog/low cloud is present, the difference between the brightness temperatures at the longwave infrared channel at $10.7 \mu \mathrm{m}$ and the shortwave-infrared channel at $3.9 \mu \mathrm{m}$ is negative during nighttime, typically between -4 and $-1 \mathrm{~K}$ depending on the droplet sizes; the smaller the droplet, the larger the difference. A number of studies have demonstrated the skill of this bispectral brightness temperature difference in fog/low-cloud detection (Bendix 2002; Ellrod 1995; Eyre et al. 1984; Lee et al. 1997; Turner et al. 1986). During daytime when scattered sunlight is present, the brightness temperature difference is positive, typically between 10 and $30 \mathrm{~K}$, which overlaps with the brightness temperature difference over cloud-free sea. 


\section{6/07/15 UTC 23:05}

(a) MODIS visible imagery

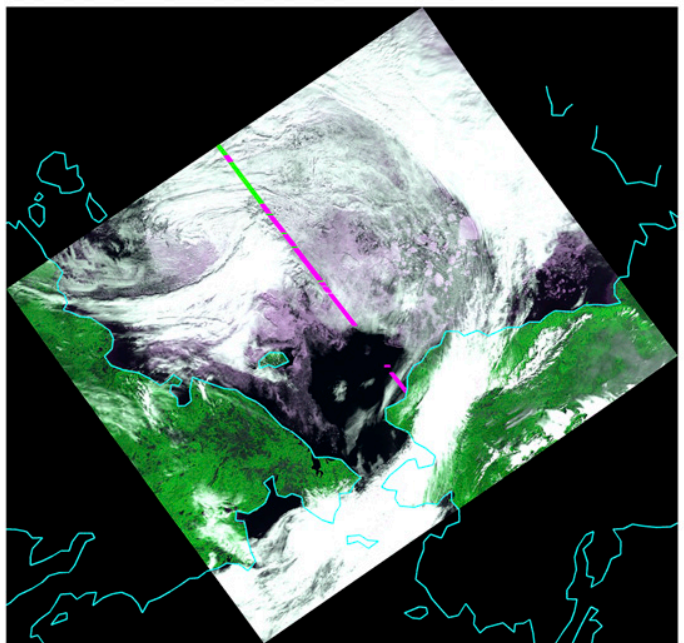

- other cloud (CALIPSO)

- fog/stratus (CALIPSO) (b) MODIS fog/stratus detected by the $\partial T$ method

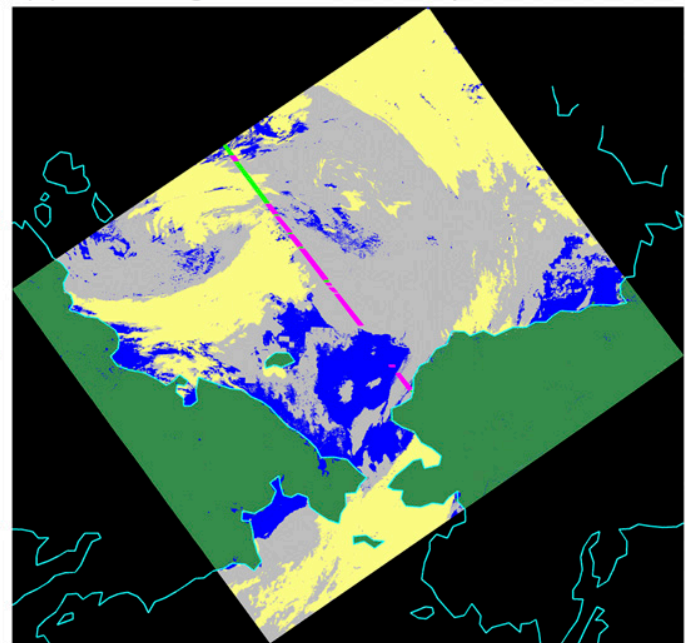

fog/stratus (MODIS)

other cloud (MODIS)

FIG. 5. (a) A visible image of the MODIS swath scan at 2305 UTC 15 July 2016. The cyan line represents the coastline. The fog detected by CALIPSO (cloud-base height $\leq 304 \mathrm{~m}$ ) along its track is overlaid as a pink line. Other types detected by CALIPSO along its track are overlaid as a green line. Note that the actual swath width of CALIPSO $(125 \mathrm{~m})$ is much narrower than the thickness of the track shown. (b) The MODIS fog product derived using the $\partial \mathrm{T}$ method. The same CALIPSO fog product shown in (a) is also overlaid for comparison. The color codes are as follows: dark green, land; blue, ocean; gray, fog; and yellow, other cloud types.

In contrast, our purpose is to detect fog based on the cloud-base information, which is not directly implied by the bispectral method. We show in Fig. 6 the bispectral brightness temperature difference $\Delta T_{B}$, defined as the brightness temperature of the MODIS channel 31 $(10.7 \mu \mathrm{m})$ minus that of channel $20(3.9 \mu \mathrm{m})$, for the collocated MODIS/CALIPSO footprints during nighttime. The MODIS channel 20 data are also obtained from the level 1 MYD021KM products. The pixels included in Fig. 6 are the same as those used in Fig. 4 that satisfy Eq. (2). The histograms of the $\Delta T_{B}$ distributions over open water and sea ice are binned into $1-\mathrm{K}$ intervals (Figs. 6a,c). The POD-FAR relation are shown in Figs. $6 \mathrm{~b}$ and $6 \mathrm{~d}$. For clarity, only the POD-FAR corresponding to $\Delta T_{B}=-4,-2,0$, and $2 \mathrm{~K}$ are shown. Over open water (Fig. 6a), the $\Delta T_{B}$ distribution for the "fog/low cloud" group is negatively skewed between -5 and $3 \mathrm{~K}$ with a peak near $-0.5 \mathrm{~K} ; \Delta T_{B}$ for "other cloud" group is distributed between -10 and $3 \mathrm{~K}$ and it is negatively skewed with a peak at $0.5 \mathrm{~K}$. The FAR is always greater than $49 \%$ (Fig. $6 \mathrm{~b}$ ), showing that $\Delta T_{B}$ cannot be used to detect fog over open water. The $\Delta T_{B}$ distributions for the two groups are very similar over sea ice (Fig. 6c), which are both negatively skewed with a peak at $0.5 \mathrm{~K}$. The resultant POD-FAR relation (Fig. 6d) shows little dependence on the $\Delta T_{B}$ threshold.
The FAR is always close to $26 \%$ regardless of the choice of the $\Delta T_{B}$ threshold.

\section{Summary}

We have developed the $\partial T$ method for the detection of fog/low cloud over the Arctic Ocean, where $\partial T$ is defined as the cloud-top temperature minus the surface temperature under the cloud. Fog/low cloud in this work is defined as a cloud object that has a base lower than $1000 \mathrm{ft}(304 \mathrm{~m})$. A fog/low-cloud event is said to be detected if $\partial T$ is greater than a threshold, which is derived using MODIS channel 31 measurements at $10.7 \mu \mathrm{m}$ and the surface temperature provided in the standard MODIS data products. Prior information of fog/low-cloud events required for defining the thresholds are obtained from a subset of collocated CALIPSO cloud-base height measurements. Since MODIS and CALIPSO have different scanning geometry and spatial resolution, the selection of the collocated MODIS/CALIPSO measurements is to ensure that both MODIS and CALIPSO have the same nadir footprints and that they are seeing the same single-layered cloud in the fields of view. Depending on the observation period (day or night) and the surface types (open water or sea ice), the $\partial T$ threshold lies between -6 and $-12 \mathrm{~K}$ over a test region in the 

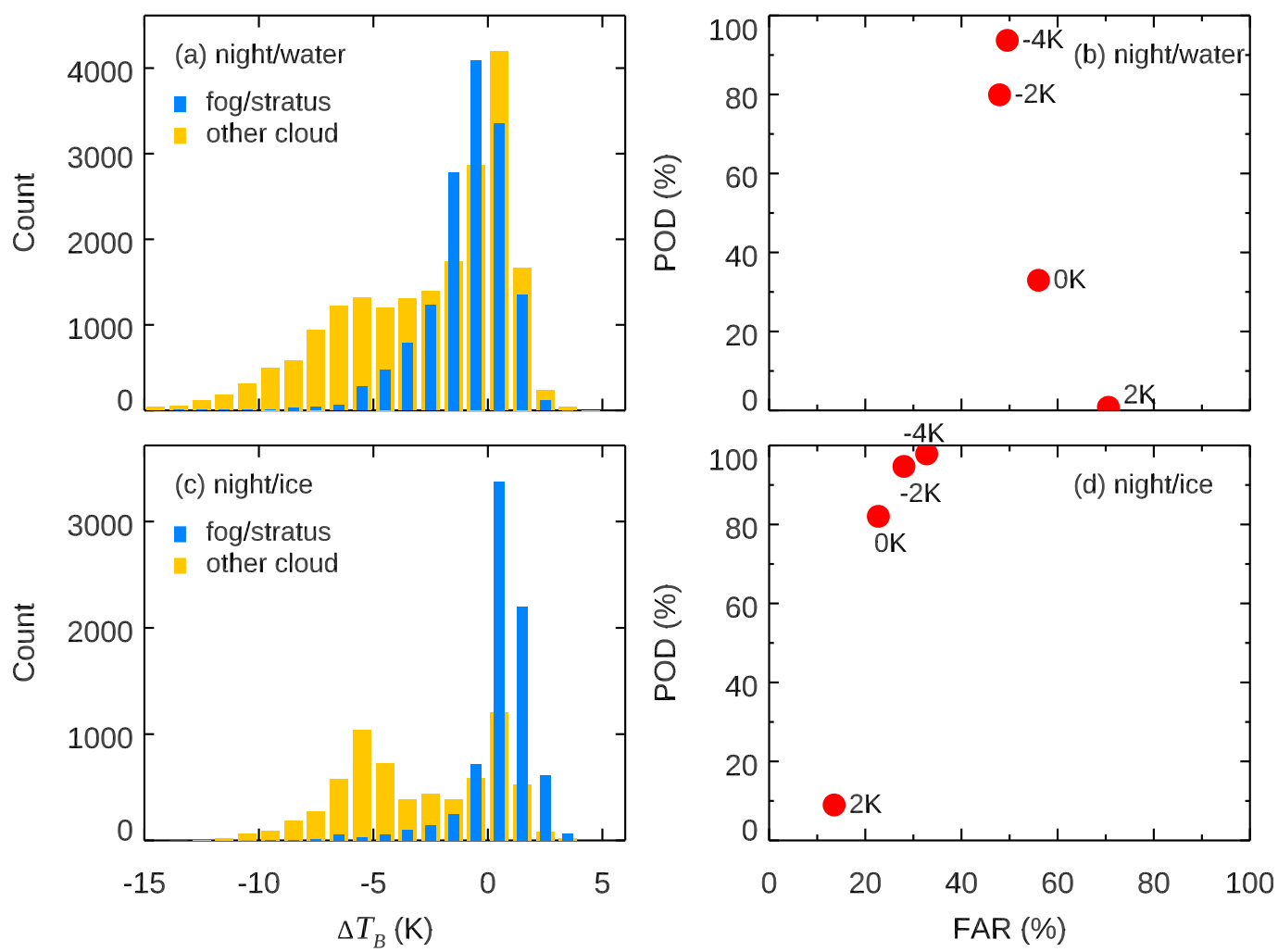

FIG. 6. The bispectral brightness temperature difference $\Delta T_{B}$, defined as the brightness temperature obtained from MODIS channel $31(10.7 \mu \mathrm{m})$ minus that obtained from MODIS channel $20(3.9 \mu \mathrm{m})$.

Chukchi Sea and the Beaufort Sea. With the collocated MODIS/CALIPSO measurements, we show that the $\partial T$ method may detect Arctic fog/low cloud over the ocean with an optimal POD of $74 \%-90 \%$ and FAR of $5 \%-17 \%$.

Acknowledgments. YL was supported by the National Key R\&D Program of China (2016YFC1402702), the National Natural Science Foundation of China (41605006), and the Shandong Provincial Natural Science Foundation of China (ZR2016DB26). KFL and KKT were supported by the Belmont Forum and the U.S. National Science Foundation through Grant NSF1536175, and by National Aeronautics and Space Administration through Grant NNX14AR40G. XC was supported by the Belmont Forum and the Natural Science Foundation of China through Grant 41561144001 and the National Key Basic Research Program of China through Grant 2015CB953900. The MODIS and CALIPSO data used in this study were obtained online (https://ladsweb.modaps.eosdis.nasa.gov/ archive/allData/6/; https://eosweb.larc.nasa.gov/project/ calipso/calipso_table).

\section{REFERENCES}

Ackerman, S. A., K. I. Strabala, W. P. Menzel, R. A. Frey, C. C. Moeller, and L. E. Gumley, 1998: Discriminating clear sky from clouds with MODIS. J. Geophys. Res., 103, 32141-32157, https://doi.org/10.1029/1998JD200032.

Baldocchi, D., and E. Waller, 2014: Winter fog is decreasing in the fruit growing region of the Central Valley of California. Geophys. Res. Lett., 41, 3251-3256, https://doi.org/10.1002/ 2014GL060018.

Bendix, J., 2002: A satellite-based climatology of fog and low-level stratus in Germany and adjacent areas. Atmos. Res., 64, 3-18, https://doi.org/10.1016/S0169-8095(02)00075-3.

— , B. Thies, J. Cermak, and T. Nauss, 2005: Ground fog detection from space based on MODIS daytime data-A feasibility study. Wea. Forecasting, 20, 989-1005, https://doi.org/ 10.1175/WAF886.1.

Cermak, J., and J. Bendix, 2007: Dynamical nighttime fog/low stratus detection based on Meteosat SEVIRI data: A feasibility study. Pure Appl. Geophys., 164, 1179-1192, https:// doi.org/10.1007/s00024-007-0213-8.

— , and —, 2011: Detecting ground fog from space-A microphysics-based approach. Int. J. Remote Sens., 32, 33453371, https://doi.org/10.1080/01431161003747505.

Cho, H.-M., and Coauthors, 2015: Frequency and causes of failed MODIS cloud property retrievals for liquid phase clouds over global oceans. J. Geophys. Res. Atmos., 120, 4132-4154, https://doi.org/10.1002/2015JD023161.

Cressey, D., 2011: Scientific challenges in the Arctic: Open water. Nature, 478, 174-177, https://doi.org/10.1038/478174a.

Cronin, T. W., and E. Tziperman, 2015: Low clouds suppress Arctic air formation and amplify high-latitude continental winter warming. Proc. Natl. Acad. Sci. USA, 112, 11490-11495, https://doi.org/10.1073/pnas.1510937112. 
Eastman, R. M., and S. G. Warren, 2010: Interannual variations of Arctic cloud types in relation to sea ice. J. Climate, 23, 4216-4232, https://doi.org/10.1175/2010JCLI3492.1.

Egli, S., B. Thies, and J. Bendix, 2018: A hybrid approach for fog retrieval based on a combination of satellite and ground truth data. Remote Sens., 10, 628, https://doi.org/10.3390/ rs10040628.

Ellrod, G. P., 1995: Advances in the detection and analysis of fog at night using GOES multispectral infrared imagery. Wea. Forecasting, 10, 606-619, https://doi.org/10.1175/ 1520-0434(1995)010<0606:AITDAA > 2.0.CO;2.

_ 2003: Estimation of low cloud base heights at night from satellite infrared and surface temperature data. Natl. Wea. Dig., 26 (1-2), 39-44.

Eyre, J. R., J. L. Brownscombe, and R. J. Allam, 1984: Detection of fog at night using Advanced Very High-Resolution Radiometer (AVHRR) imagery. Meteor. Mag., 113, 266-271.

Gao, S., W. Wu, L. Zhu, G. Fu, and B. Huang, 2009: Detection of nighttime sea fog/stratus over the Huanghai Sea using MTSAT-1R IR data. Acta Oceanol. Sin., 28, 23-35.

Gultepe, I., M. Pagowski, and J. Reid, 2007: A satellite-based fog detection scheme using screen air temperature. Wea. Forecasting, 22, 444-456, https://doi.org/10.1175/WAF1011.1.

Holz, R. E., S. A. Ackerman, F. W. Nagle, R. Frey, S. Dutcher, R. E. Kuehn, M. A. Vaughan, and B. Baum, 2008: Global Moderate Resolution Imaging Spectroradiometer (MODIS) cloud detection and height evaluation using CALIOP. J. Geophys. Res., 113, D00A19, https://doi.org/10.1029/ 2008JD009837.

Hunt, G. E., 1973: Radiative properties of terrestrial clouds at visible and infrared thermal window wavelengths. Quart. J. Roy. Meteor. Soc., 99, 346-369, https://doi.org/10.1002/ qj.49709942013.

Intrieri, J. M., C. W. Fairall, M. D. Shupe, P. O. G. Persson, E. L. Andreas, P. S. Guest, and R. E. Moritz, 2002: An annual cycle of Arctic surface cloud forcing at SHEBA. J. Geophys. Res., 107, 8039, https://doi.org/10.1029/2000JC000439.

Kay, J. E., and A. Gettelman, 2009: Cloud influence on and response to seasonal Arctic sea ice loss. J. Geophys. Res., 114, D18204, https://doi.org/10.1029/2009JD011773.

Koračin, D., C. E. Dorman, J. M. Lewis, J. G. Hudson, E. M. Wilcox, and A. Torregrosa, 2014: Marine fog: A review. Atmos. Res., 143, 142-175, https://doi.org/10.1016/ j.atmosres.2013.12.012.

Lasserre, F., and S. Pelletier, 2011: Polar super seaways? Maritime transport in the Arctic: An analysis of shipowners' intentions. J. Transp. Geogr., 19, 1465-1473, https://doi.org/10.1016/ j.jtrangeo.2011.08.006

Lee, T. F., F. J. Turk, and K. Richardson, 1997: Stratus and fog products using GOES-8-9 3.9- $\mu \mathrm{m}$ data. Wea. Forecasting, 12, 664-677, https://doi.org/10.1175/1520-0434(1997)012<0664: SAFPUG $>2.0 . \mathrm{CO} ; 2$

Liu, Y., and J. R. Key, 2014: Less winter cloud aids summer 2013 Arctic sea ice return from 2012 minimum. Environ. Res. Lett., 9, 044002, https://doi.org/10.1088/1748-9326/9/4/ 044002.

Menzel, W. P., and Coauthors, 2008: MODIS global cloud-top pressure and amount estimation: Algorithm description and results. J. Appl. Meteor. Climatol., 47, 1175-1198, https:// doi.org/10.1175/2007JAMC1705.1.

Morrison, A. L., J. E. Kay, H. Chepfer, R. Guzman, and V. Yettella, 2018: Isolating the liquid cloud response to recent Arctic sea ice variability using spaceborne lidar observations.
J. Geophys. Res. Atmos., 123, 473-490, https://doi.org/10.1002/ 2017JD027248.

Palm, S. P., S. T. Strey, J. Spinhirne, and T. Markus, 2010: Influence of Arctic sea ice extent on polar cloud fraction and vertical structure and implications for regional climate. J. Geophys. Res., 115, D21209, https://doi.org/10.1029/ 2010JD013900.

Reynolds, R. W., T. M. Smith, C. Liu, D. B. Chelton, K. S. Casey, and M. G. Schlax, 2007: Daily high-resolution-blended analyses for sea surface temperature. J. Climate, 20, 5473-5496, https://doi.org/10.1175/2007JCLI1824.1.

Schaefer, J. T., 1990: The critical success index as an indicator of warning skill. Wea. Forecasting, 5, 570-575, https://doi.org/ 10.1175/1520-0434(1990)005<0570:TCSIAA $>2.0 . C O ; 2$.

Schweiger, A. J., R. W. Lindsay, S. Vavrus, and J. A. Francis, 2008: Relationships between Arctic sea ice and clouds during autumn. J. Climate, 21, 4799-4810, https://doi.org/10.1175/ 2008JCLI2156.1.

Shupe, M. D., and J. M. Intrieri, 2004: Cloud radiative forcing of the Arctic surface: The influence of cloud properties, surface albedo, and solar zenith angle. J. Climate, 17, 616-628, https://doi.org/ 10.1175/1520-0442(2004)017<0616:CRFOTA > 2.0.CO;2.

Smith, L. C., and S. R. Stephenson, 2013: New trans-Arctic shipping routes navigable by midcentury. Proc. Natl. Acad. Sci. USA, 110, E1191-E1195, https://doi.org/10.1073/pnas.1214212110.

Stephenson, S. R., L. C. Smith, and J. A. Agnew, 2011: Divergent long-term trajectories of human access to the Arctic. Nat. Climate Change, 1, 156-160, https://doi.org/ 10.1038/nclimate1120.

Turner, J., R. J. Allam, and D. R. Maine, 1986: A case-study of the detection of fog at night using channels 3 and 4 on the Advanced Very High-Resolution Radiometer (AVHRR). Meteor. Mag., 115, 285-290.

Vavrus, S., D. E. Waliser, A. Schweiger, and J. Francis, 2009: Simulations of 20th and 21st century Arctic cloud amount in the global climate models assessed in the IPCC AR4. Climate Dyn., 33, 1099-1115, https://doi.org/10.1007/s00382-008-0475-6.

Wang, X., and J. R. Key, 2005: Arctic surface, cloud, and radiation properties based on the AVHRR Polar Pathfinder dataset. Part II: Recent trends. J. Climate, 18, 2575-2593, https:// doi.org/10.1175/JCLI3439.1.

Warren, S. G., R. M. Eastman, and C. J. Hahn, 2007: A survey of changes in cloud cover and cloud types over land from surface observations, 1971-96. J. Climate, 20, 717-738, https://doi.org/ 10.1175/JCLI4031.1.

Wilcox, E. M., 2017: Multi-spectral remote sensing of sea fog with simultaneous passive infrared and microwave sensors. Marine Fog: Challenges and Advancements in Observations, Modeling, and Forecasting, D. Koračin and C. E. Dorman, Eds., Springer, 511-526.

WMO, 2005: Aerodrome reports and forecasts: A user's handbook to the codes. 4th ed. World Meteorological Organization Rep., $75 \mathrm{pp}$.

Wu, D., B. Lu, T. Zhang, and F. Yan, 2015: A method of detecting sea fogs using CALIOP data and its application to improve MODIS-based sea fog detection. J. Quant. Spectrosc. Radiat. Trans., 153, 88-94, https://doi.org/10.1016/j.jqsrt.2014.09.021.

Wu, X., and S. Li, 2014: Automatic sea fog detection over Chinese adjacent oceans using Terra/MODIS data. Int. J. Remote Sens., 35, 7430-7457, https://doi.org/10.1080/ 01431161.2014.968685.

Yamanouchi, T., and S. Kawaguchi, 1992: Cloud distribution in the Antarctic from AVHRR data and radiation measurements at 
the surface. Int. J. Remote Sens., 13, 111-127, https://doi.org/ 10.1080/01431169208904029.

Yi, L., S. Zhang, B. Thies, X. Shi, K. Trachte, and J. Bendix, 2015: Spatio-temporal detection of fog and low stratus top heights over the Yellow Sea with geostationary satellite data as a precondition for ground fog detection-A feasibility study. Atmos. Res., 151, 212-223, https://doi.org/10.1016/ j.atmosres.2014.03.020.
_ B. Thies, S. Zhang, X. Shi, and J. Bendix, 2016: Optical thickness and effective radius retrievals of low stratus and fog from MTSAT daytime data as a prerequisite for Yellow Sea fog detection. Remote Sens., 8, 8, https://doi.org/10.3390/rs8010008.

Zhang, S., and L. Yi, 2013: A comprehensive dynamic threshold algorithm for daytime sea fog retrieval over the Chinese adjacent seas. Pure Appl. Geophys., 170, 1931-1944, https:// doi.org/10.1007/s00024-013-0641-6. 